\title{
Trends of Malaria Morbidity and Mortality from 2010 to 2017 in Bale Zone, Ethiopia: Analysis of Surveillance Data
}

This article was published in the following Dove Press journal: Infection and Drug Resistance

\author{
Falaho Sani Kalil iD ' \\ Mohammed Hasen Bedaso iD ${ }^{2}$ \\ Shukri Kabeta Wario ${ }^{3}$ \\ 'Negelle Borena Health Science College, \\ Negelle Borena, Oromia, Ethiopia; \\ ${ }^{2}$ Public Health Emergency Management, \\ East Bale Zonal Health Office, Ginnir, \\ Ethiopia; ${ }^{3}$ Public Health Emergency \\ Management, Bale Zonal Health Office, \\ Robe, Ethiopia
}

Background: Malaria is the major public health problem in sub-Saharan Africa, including Ethiopia. Ongoing malaria surveillance data analysis is useful for assessing incidences, trends over time, and evaluating the effectiveness of malaria prevention and control programs.

Objective: To describe trends in malaria morbidity and mortality from 2010 to 2017 using surveillance data in Bale zone, Southeast Ethiopia.

Methods: A retrospective study was conducted. Data were extracted from a public health emergency management surveillance database of Bale zonal health department. Data were entered into Microsoft office Excels worksheet 2016 and analyzed using Epi info version 7.2 software. Descriptive statistics was employed to calculate frequencies and percentages of malaria cases, trends of malaria transmission in terms of years, plasmodium species, gender, age, geographical and seasonal distribution. Malaria morbidity were assessed using the incidence rate of malaria cases per 1,000 population at risk and analyzed by year.

Results: A total of 16,465 malaria cases were reported over 8 years. Of these, 10,986 $(66.7 \%)$ were confirmed cases by microscopy/rapid diagnostic test. The majority of the cases, $82.2 \%$, were reported among the $>5$ years age group and $62.9 \%$ were males. The overall 8 years average annual incidence was 3.1 cases/1,000 population at risk. There was an increase in incidence by $26 \%$ between $2010-2012$, then a fall by $85 \%$ from $2012-2014$, with another increaseby $52 \%$ from 2014-2017. The majority of the confirmed cases $(81.5 \%)$ were due to Plasmodium falciparum species. The overall 8 years average annual death rate from malaria was $0.15 / 100,000$ population.

Conclusion: Even though a substantial reduction in morbidity and mortality of malaria was achieved, the possibility of observing severe cases was higher in the study area. Hence, the prevention and control program should be sustained and adjusted to address Plasmodium falciparum species.

Keywords: malaria, incidence, trend, morbidity, mortality, Bale zone, Southeast Ethiopia

\section{Background}

Malaria is caused by any of the five species of Plasmodium (P. falciparum, $P$. vivax, P. ovale, P. Malariae, and P. knowlesi in Asia) protozoan parasite infection. It is the most important of the life-threatening parasitic diseases of humans with enormous medical, economical, and emotional impacts in the world. ${ }^{1}$ In the year 2016, an estimated 216 million cases of malaria occurred worldwide compared with 237 million cases in 2010, and 211 million cases in 2015. Most malaria cases in
Correspondence: Falaho Sani Kali Tel +251913963292

Email falahsani@gmail.com

Infection and Drug Resistance 2020:13 4379-4387 
2016 were in the WHO (World Health Organization) African region (90\%). Of the 91 countries reporting indigenous malaria cases in 2016, 15 countries - all in subSaharan Africa (SSA), carried $80 \%$ of the global malaria burden. ${ }^{2}$ The incidence of malaria is decreased by $18 \%$ globally, from 76 to 63 cases per 1,000 population at risk between the years 2010 and 2016. Plasmodium falciparum is the most prevalent malaria parasite in SSA, accounting for $99 \%$ of estimated malaria cases in $2016 .{ }^{2}$ In 2016 , there were an estimated 445,000 deaths from malaria globally, compared to 446,000 in 2015 . The majority of deaths (91\%) from malaria in 2016 were from the WHO African region. ${ }^{2}$

Beyond the huge health consequence, malaria imposes a substantial economic burden on individuals, households, and the entire economy. ${ }^{3}$ Malaria disease has been found to reduce the potential economic growth rate by $1.3 \%$ per year in some African countries as a single disease. According to Gallup and Sachs' ${ }^{4}$ study, malaria and poverty are intimately connected at the macro-level, in which malaria is the main contributor to poverty.

Approximately $60 \%$ of Ethiopia's population live in malaria areas, and $68 \%$ of the country's landmass is favorable for malaria transmission (below 2,000 meters above sea level), with malaria primarily associated with altitude and rainfall. ${ }^{5,6}$ According to EPHI (Ethiopian Public Health Institute) annual performance report, the total number of laboratory-confirmed plus clinical malaria cases were $2,174,707$ in $2014 / 2015$. Of these, $1,867,059$ (85.9\%) were confirmed by either microscopy or Rapid Diagnostic Tests (RDTs) and 1,188,627 (63.7\%) were Plasmodium falciparum and 678,432 (36.3\%) were P. vivax. ${ }^{6}$ A malaria indicator survey of Ethiopia conducted in 2015 reported that the national proportion of P. falciparum was $81.6 \%$ and the proportion of $P$. vivax was $8.0 \%$, while the malaria indicator survey data of Oromia region indicated $87.5 \%$ and $12.5 \%$ proportions of P. falciparum and P. vivax, respectively. ${ }^{5}$

Bale zone has great geographical diversity; its topographic features range from the highest peak at Bale mountain, 4,288 meters above sea level, down to the lowland area, at 331 meters. The topography of the majority of the districts $(80 \%)$ in Bale zone is located at an altitude below 2,000 meters above sea level, with $64 \%$ of zonal land mass being lowlands, $22 \%$ midlands, and only $14 \%$ highlands. Almost half of Bale zone's surface area is malaria endemic (altitude below 2,000 meters above sea level), which means $46 \%$ of the population are at risk for malaria. Malaria is one of the major public health problems in Bale zone, and has been reported as one of the ten top diseases in the health facilities. The peak of malaria incidence in Bale zone occurs from October to December, followed by April to June (Unpublished administrative data of Bale zonal health department). Peak malaria transmission occurs between September and December in most parts of Ethiopia, after the main rainy season from June to August. In addition, some areas experience a second minor malaria transmission period from April to June, following a short rainy season from February to March. ${ }^{6}$

Ethiopia proposed a national strategic goal for the prevention, control, and elimination of malaria. The proposed objectives from 2017 to 2020 were reducing the number of malaria cases by $40 \%$ from baseline of 2016 by the year 2020, achieving near zero malaria deaths (no more than one confirmed malaria death per 100,000 population at risk), and eliminating malaria from Ethiopia by $2030 .^{6}$

In order to monitor the trends of malaria morbidity and mortality, an unusual increase and decreases of the disease and evaluating the effectiveness of malaria prevention and control programs, the ongoing analysis and interpretation of the surveillance data are necessary. This information is also needed to determine the allocation of public health resources and personnel. ${ }^{7}$ Therefore, the focus of this study was to describe the trends in morbidity and mortality of malaria from 2010 to 2017 using the surveillance data in Bale zonal health department, Southeast Ethiopia.

\section{Methods}

\section{Study Setting}

The study was conducted in Bale zone of the Oromia regional state, Southeast Ethiopia. Robe town, the capital of Bale zone, is located 445 kilometers Southwest of Addis Ababa, the capital of Ethiopia. The zone lies at between $39^{\circ} 59^{\prime}$ to $39^{\circ} 98^{\prime}$ East longitude and $7^{\circ} 0^{\prime}$ to $7^{\circ}$ $01^{\prime}$ North latitude. The area has a mean temperature of $15.6^{\circ} \mathrm{C}$ and an annual rainfall of 1,272 milliliters (Ethiopian Meteorology Agency, unpublished data). Bale zone has an estimated population of $1,854,699$, of whom 944,042 are males and $86.4 \%$ reside in rural areas. ${ }^{8}$ According to the administrative report, the total area of the zone is estimated to be $67,329.59$ square kilometers and divided into 15 administrative districts (woredas) and 386 kebeles (smallest administrative 
unit). There are five public hospitals, 85 health centers, and 380 health posts in Bale zone, and malaria diagnosis services have been provided in the public and private health facilities.

\section{Study Design and Population}

A retrospective study was conducted to determine the trends of malaria morbidity and mortality using the registered cases of malaria from 2010 to 2017 in Bale zone. The target populations for the study were all malaria suspected individuals who had visited the health facilities in Bale zone from January 2010 to December 2017.

\section{Cases and Operational Definitions}

Suspected malaria: Any person with a fever or fever with headache, chills, rigor, back pain, sweats, myalgia, nausea, and vomiting diagnosed clinically as malaria. ${ }^{9}$

Confirmed malaria: Any suspected cases of malaria confirmed by microscopy or Rapid Diagnostic Test (RDT) for plasmodium parasite.

Clinically diagnosed malaria cases: A suspected cases of malaria based on clinical manifestation without confirmation by either microscopy or rapid diagnostic test. ${ }^{2}$

Clinical and confirmed case of malaria: The sum of malaria cases diagnosed clinically and the cases confirmed by microscopy or RDT. ${ }^{9}$

Malaria Test positivity rate: The percentage of malaria cases confirmed by microscopy/RDT out of the cases tested/examined.

Malaria incidence: The number of malaria cases per 1,000 population at risk in the catchment area. ${ }^{2}$

Malaria mortality rate (death rate): Deaths from malaria cases per 100,000 population at risk in the

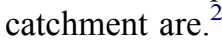

Malarious area: Area which is located at an altitude level below 2,000 meters above sea level. ${ }^{5}$

Partially malarious district: A district in which its topography is partially located at an altitude level less than 2,000 meters and some kebeles of the district found at an altitude more than 2,000 meters above sea level. ${ }^{5}$

\section{Source of Data and Procedure}

Eight years (2010-2017) records of malaria data were extracted from the public health emergency management surveillance database of Bale zonal health department. Data extracted included information such as: each district's clinically diagnosed malaria cases by months, monthly confirmed malaria cases (microscopy/RDT) by districts, suspected cases tested by microscopy/RDT, malaria cases by Plasmodium parasite, malaria cases by season (month), deaths from malaria, treatment status such as inpatient and outpatient, and socio-demographic status such as gender and age group. Data were extracted by an experienced field epidemiologist from the public health emergency department of Bale zone. Data related to the population at risk in each district (woreda) were collected from the malaria department of Bale zone.

\section{Data Quality Control}

The completeness of the malaria surveillance data was checked before data collection to ensure the quality of data. Data were checked by year from 2010 to 2017, by woredas in Bale zone and for completeness of all variables. Then, a data collection format sheet was prepared using Microsoft Excel and used for data recording. The data collector was adequately trained for 2 days prior to data extraction. The overall process of data extraction was followed up by the principal investigator and data were checked for accuracy, completeness, and consistency before analysis. Any data that were not properly documented and not completely registered were excluded from analysis.

\section{Data Analysis Procedure}

Data were entered into a Microsoft office Excel worksheet 2016 and analyzed using Epi info version 7.2 software. Descriptive statistics were employed to calculate frequencies and percentages of overall malaria cases, trends of malaria transmission in terms of years, Plasmodium species, gender, age, geographical distribution (by woredas), and distribution by seasons (months). Malaria morbidity was assessed using the incidence rate of malaria cases per 1,000 population at risk and analyzed by years. Trends of microscopy/RDT confirmed vs clinical malaria cases were summarized by years. Test positivity rates of malaria were calculated and described by positivity percentage by years. The registered populations at risk by woredas in Bale zone were used to calculate the incidence and death rate, and analyzed by years. Findings were summarized using tables, line graphs, and a bar charts.

\section{Results}

\section{Trends in Malaria Morbidity and Mortality from 2010 to 2017}

Within an 8-year period (2010-2017), a total of 62,392 suspected malaria cases were reported from Bale zone. 
Out of these, 10,986 (66.7\%) were confirmed malaria cases either by microscopy or RDT and 5,479 (33.3\%) were clinically diagnosed, making a total of 16,465 malaria cases during an 8 -years period. The majority of the reported cases $(15,872,96.4 \%)$ were treated in an outpatient department of health facilities, while only 588 cases $(3.6 \%)$ were admitted.

The highest number of malaria cases $(4,184,25.4 \%)$ were observed in 2012, followed by 3,410 (20.7\%) in 2011. The number of malaria cases (laboratory confirmed and clinically diagnosed) sharply increased from 2,446 in 2010 to 4,184 in 2012. However, there was a substantial decline in the number of malaria cases, to 1,931 and 722 in 2013 and 2014, respectively. Then, there was a progressive increase from 1,135 cases in 2015 to 1,540 in 2017 (Table 1).

The overall mean ( \pm standard deviation) annual incidence of malaria from 2010 to 2017 was 3.1 cases $( \pm 2.1)$ per 1,000 population at risk. The incidence of malaria measured in 2010 was 4.8 per 1,000 population, then increased to 5.5 and 6.1 per 1,000 population in 2011 and 2012, respectively. The incidence of malaria sharply declined to 0.91 per 1,000 population from 2013-2014. There was an increase in incidence of malaria again to 1.9 per 1,000 population between 2015 and 2017. There was an increase in incidence of malaria by $26 \%$ between 2010 and 2012, then the highest decline by 85\%, was observed between 2012 and 2014. Then, the incidence of malaria were increased by $52 \%$ between 2014 and 2017 (Figure 1).

A total of nine deaths from malaria, with a mean annual death rate of $0.15 / 100,000$ populations per year registered during the 8 -years period. The overall malaria case fatality ratio was $0.05 \%$, while the laboratory confirmed (microscopy/RDT) malaria case fatality ratio was
$0.08 \%$. The highest number of deaths from malaria (six deaths) were reported in 2017 , with an annual death rate of 0.72 deaths per 100,000 population. There were no deaths reported in the year 2010, and between 2013 and 2016 (Table 1).

\section{Morbidity of Malaria by Plasmodium Parasite}

The dominant parasite of confirmed malaria cases during the study period was Plasmodium falciparum, which accounted for 8,950 (81.5\%) of cases, while $P$. vivax constituted 2,036 $(18.5 \%)$ cases. There was temporal fluctuation in the number of confirmed malaria cases by microscopy/RD, which increased from 1,280 in 2010 to 2,977 cases in 2012, then progressively fell to 599 in 2014 . However, the number of confirmed malaria cases substantially increased from 599 in 2014 to 1,394 cases in 2017 (Figure 2). The Plasmodium falciparum was predominately reported throughout the study period from 2010 to 2017 (Figure 2). There was no report other than these two species. The overall mean $( \pm \mathrm{SD})$ annual incidence of confirmed malaria during the 8 years was 2.0 cases $( \pm 1.2)$ per 1,000 population at risk. The trends in annual incidence of confirmed vs clinically diagnosed malaria cases is indicated in Figure 1.

The trends in Test Positivity Rate (TPR) of malaria are presented in Figure 3. The overall test positivity rate of Plasmodium parasite was $17 \%$ (10,986 cases/62,392 examined). The highest TPR of malaria (35.4\%) were observed in 2017 , followed by $26.6 \%$ in 2011 and $21 \%$ in 2012 . There were temporal decline in trends of examination of suspected malaria cases from 2012 to 2017, with the highest number of examinations observed in the year 2012 (12,161 cases), while the lowest examination of suspected cases was observed in the year 2017 (3,938 cases).

Table I Malaria Incidence and Death Rate from 2010 to 2017 in Bale Zone, Southeast Ethiopia

\begin{tabular}{|l|l|l|l|l|l|l|}
\hline Year & $\begin{array}{l}\text { Population at } \\
\text { Risk }\end{array}$ & $\begin{array}{l}\text { No. of Cases } \\
\text { (\%) }\end{array}$ & $\begin{array}{l}\text { No. of Deaths } \\
\text { (\%) }\end{array}$ & $\begin{array}{l}\text { Incidence Rate per I,000 } \\
\text { Population }\end{array}$ & $\begin{array}{l}\text { Death Rate per I00,000 } \\
\text { Population }\end{array}$ & $\begin{array}{l}\text { CFR in } \\
\%\end{array}$ \\
\hline 2010 & 504,483 & $2,446(14.9)$ & 0 & 4.85 & 0.00 & 0.00 \\
2011 & 621,779 & $3,410(20.7)$ & $1(11)$ & 5.48 & 0.16 & 0.03 \\
2012 & 682,240 & $4,184(25.4)$ & $2(22)$ & 6.13 & 0.29 & 0.00 \\
2013 & 775,529 & $1,931(11.7)$ & 0 & 2.49 & 0.00 & 0.00 \\
2014 & 791,208 & $722(4.4)$ & 0 & 0.91 & 0.00 & 0.00 \\
2015 & 788,595 & $1,135(6.9)$ & 0 & 1.44 & 0.00 & 0.00 \\
2016 & 810,987 & $1,097(6.7)$ & 0 & 1.35 & 0.72 & 0.00 \\
2017 & 831,056 & $1,540(9.4)$ & $6(67)$ & 1.85 & 0.39 \\
\hline
\end{tabular}

Abbreviation: CFR, case fatality rate. 


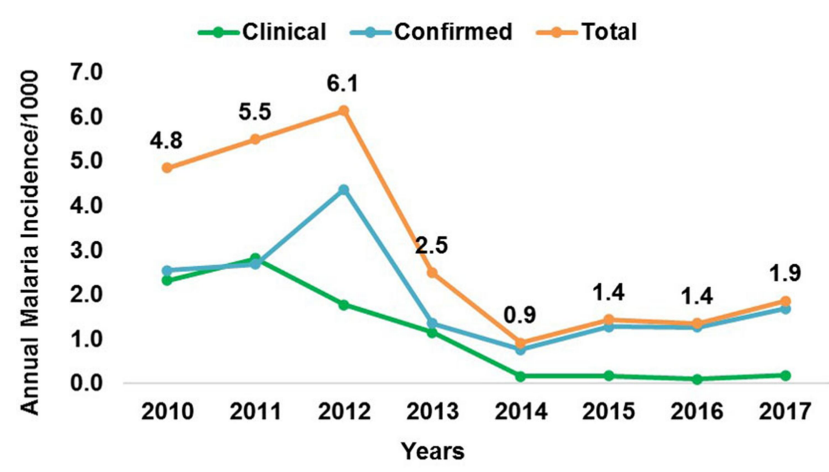

Figure I Trends of annual incidence of malaria from 2010 to 2017 in Bale zone, Southeast Ethiopia.

\section{Geographical Distribution of Malaria Morbidity and Mortality}

From a total of 15 woredas and 386 kebeles of Bale zone, $12(80 \%)$ woredas and $196(51 \%)$ kebeles were partially or completely malarious, with about $46 \%$ of the total population residing in these areas. The distribution of malaria cases varies among woredas. The highest cases of malaria during this 8 -years period were reported from Dellomena woreda (4,612 cases), followed by Ginnir, Harenabuluk, and Sawena woreda $(2,142$, 1,354 , and 1,312 , respectively). The mean annual incidence of malaria in the woredas has a different magnitude, from the lowest in Rayitu woreda, 0.41/1,000 population, to the highest in Harenabuluk woreda, 14.76/1,000 population (Figure 4). Plasmodium falciparum was the dominant species in the majority of Bale zone woredas (13/15 woredas), while the dominant species in Agarfa and Goro woreda is $P$. vivax, which accounts for $58.6 \%$ and $52.6 \%$ of the confirmed cases, respectively.

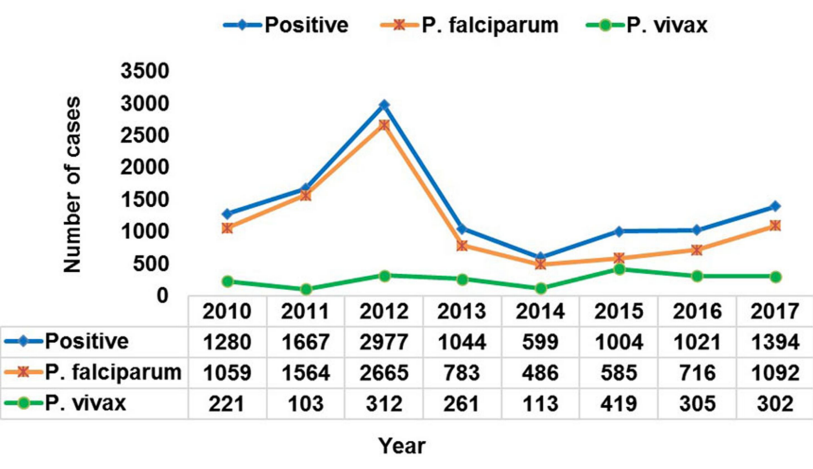

Figure 2 Trends of malaria cases by plasmodium parasite from 2010 to 2017 in Bale zone, Southeast Ethiopia.

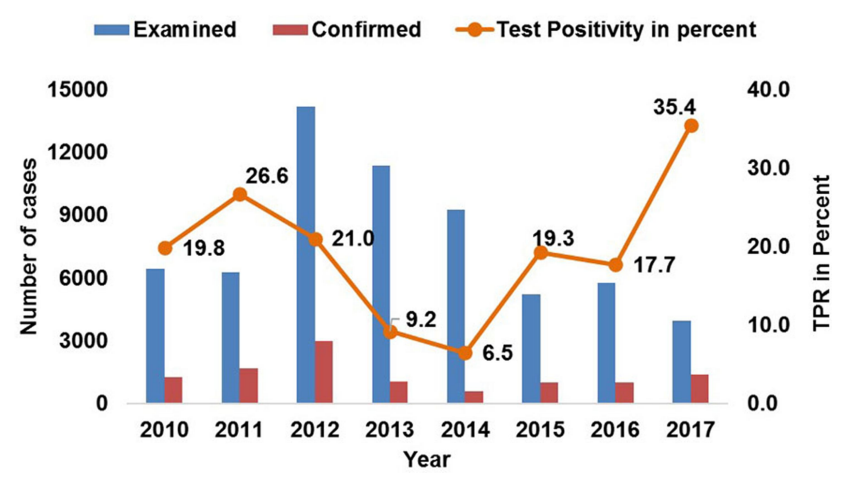

Figure 3 Trends of test positivity rate of confirmed malaria cases from 2010 to 2017 in Bale zone, Southeast Ethiopia.

\section{Morbidity of Malaria by Gender and Age Group}

Out of 16,465 malaria cases recorded in 8 years, 10,356 $(62.9 \%)$ and $6,109(37.1 \%)$ were reported in males and females, respectively. The mean annual incidence of malaria for males was 4.12 cases per 1,000 population, while for females it was 3.61 per 1,000 population, as shown in Table 2. For each year, the numbers of malaria cases among males were higher than that of females.

Regarding morbidity of malaria by age group, the majority of the cases $(13,535,82.2 \%)$ were reported among the above 5 years age group, while 2,930 (17.8\%) cases were observed in under-5 years children. Trends in annual incidence of malaria by age group are shown in Figure 5. The mean annual incidence of malaria in the under 5 and above 5 years age groups was 3.12/1,000 children and 3.06 cases per 1,000 population respectively, as shown in Table 2. The highest annual incidence of 6.5/ 1,000 in children under 5 years and 6.5/1,000 in those above 5 years was reported in 2012 .

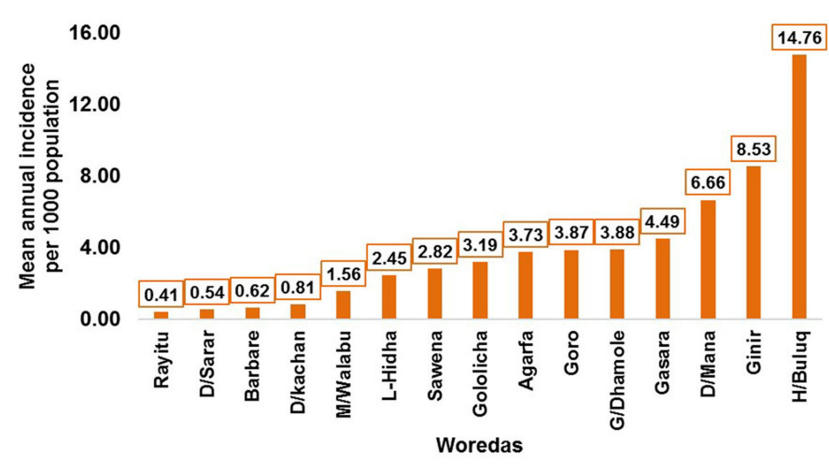

Figure 4 Mean annual incidence of malaria by Woredas/districts in Bale zone from 2010 to 2017, Southeast Ethiopia. 
Table 2 Distribution of Malaria Cases and Incidence Rate by Gender and Age from 2010 to 2017 in Bale Zone, Southeast Ethiopia

\begin{tabular}{|l|l|l|l|}
\hline Variables & Category & Number (\%) & $\begin{array}{l}\text { Mean Annual } \\
\text { Malaria Incidence } \\
\text { per I,000 } \\
\text { Population }\end{array}$ \\
\hline \multirow{2}{*}{ Gender } & Male & $10,356(62.9)$ & 4.12 \\
& Female & $6,109(37.1)$ & 3.61 \\
& $<5$ years & $2,930(17.8)$ & 3.12 \\
& Above 5 & $13,535(82.2)$ & 3.05 \\
& years & & \\
\hline
\end{tabular}

\section{Seasonal Distribution of Malaria}

The seasonal distribution of malaria cases is presented in Figure 6. Though malaria occurred in all seasons, the number of cases had a fluctuating trend across the four seasons over the last 8 years. The highest cases of malaria $(31.6 \%)$ were observed during spring (SeptemberNovember), while the lowest cases (20.1\%) were during autumn (March-May) and June. The peak in malaria cases occurred in October and November, which is harvesting season after the main rainfall. The second peak of malaria cases was observed between June and July following a short rainy season in March-April.

\section{Discussion}

Ethiopia has shown remarkable progress in achieving malaria-related Millennium Development Goal (MDG) targets, as evidenced by reduced prevalence and death rates, as well as an increase in the proportion of the population using effective malaria prevention and control measures in malaria prone areas. This study focused on describing the trends of malaria morbidity and mortality

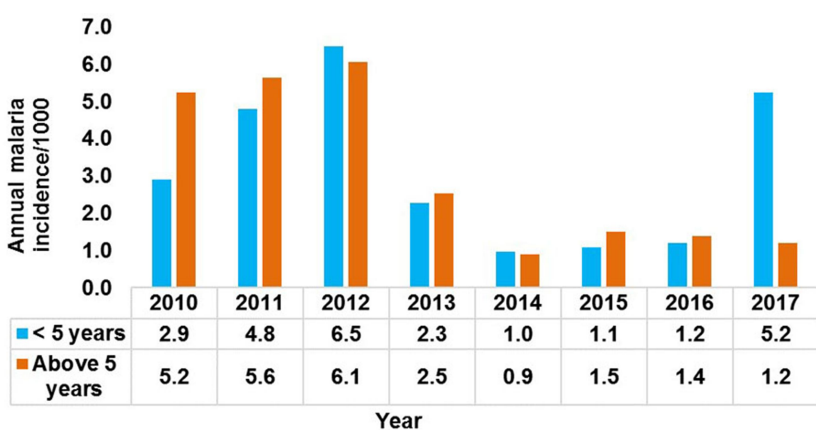

Figure 5 Annual incidence of malaria by age group from 2010 to 2017 in Bale zone, Southeast Ethiopia. from 2010 to 2017 using surveillance data of malaria in Bale zone.

The findings of this study showed that there was a decline in morbidity of malaria cases by $63.2 \%$ between 2012 and 2017, and a total 37\% decline between 2010 and 2017. Similarly, a $58 \%$ decline in trends of malaria cases was reported according to a key malaria indicators report through routine surveillance systems between 2011 and 2016, and a 52\% decline between 2010 and 2016 according to a WHO malaria report of 2017., ${ }^{2,6}$ However, an increase in malaria cases was observed during the first 3 years of the study period (2010-2012) by $71 \%$, which is possibly due to varying climatic feature and the unstable nature of malaria transmission. ${ }^{10,11}$ This could be due to low coverage of insecticide treated net and Indoor Residual Spray (IRS) sprayed in the past 12 months in Oromia region, according to the 2011 Malaria Indicator Survey (MIS) report. $^{12}$ This is supported by the 2011 report of Ethiopia MIS, which shows the low Insecticide Treated bed-Net (ITN) coverage among pregnant women (65\%) and under five children (55\%) in Oromia region compared to the 2015 MIS report, which is $81 \%$ and $75 \%$, respectively. ${ }^{5,12}$

The mean annual incidence of malaria during the study period was 3.1 cases $/ 1,000$ population at risk and all 8 -years incidences were below five cases/1,000 population at risk except in 2011 and 2012. The result of the study is by far lower than the national annual incidence rates of malaria in 2014, 2015, and 2016 which were 54.2, 45.7, and $40.3 / 1,000$ population at risk according to a WHO malaria report of $2017 .^{2}$ The incidence of malaria was decreased by $69.8 \%$ between 2012 and 2017 years, which is supported by national reduction of malaria incidence by $58.6 \%$ between 2010 and $2016 .^{2}$ The decline in incidence is explained by variation between regional figures and within the region. ${ }^{5}$ On the other hand, a substantial decline in the incidence of malaria is possibly due to the implementation of malaria prevention strategies at the community level, and as a result of high coverage of Long Lasting Insecticide treated Net (LLIN) (from $44 \%$ in 2011 to $58 \%$ in 2015) and sprayed IRS in the past 12 months (from $43 \%$ in 2011 to $67 \%$ in 2015) in Oromia region. ${ }^{5,12}$ The significant decline is also due to increased knowledge of women about malaria prevention and ITN high coverage among pregnant women and children under 5 years in Oromia region according to 2015 MIS, which were $81 \%$ and $75 \%$, respectively. 5,12 This finding is indicative of the achievements of MDG target set for 2014/15 


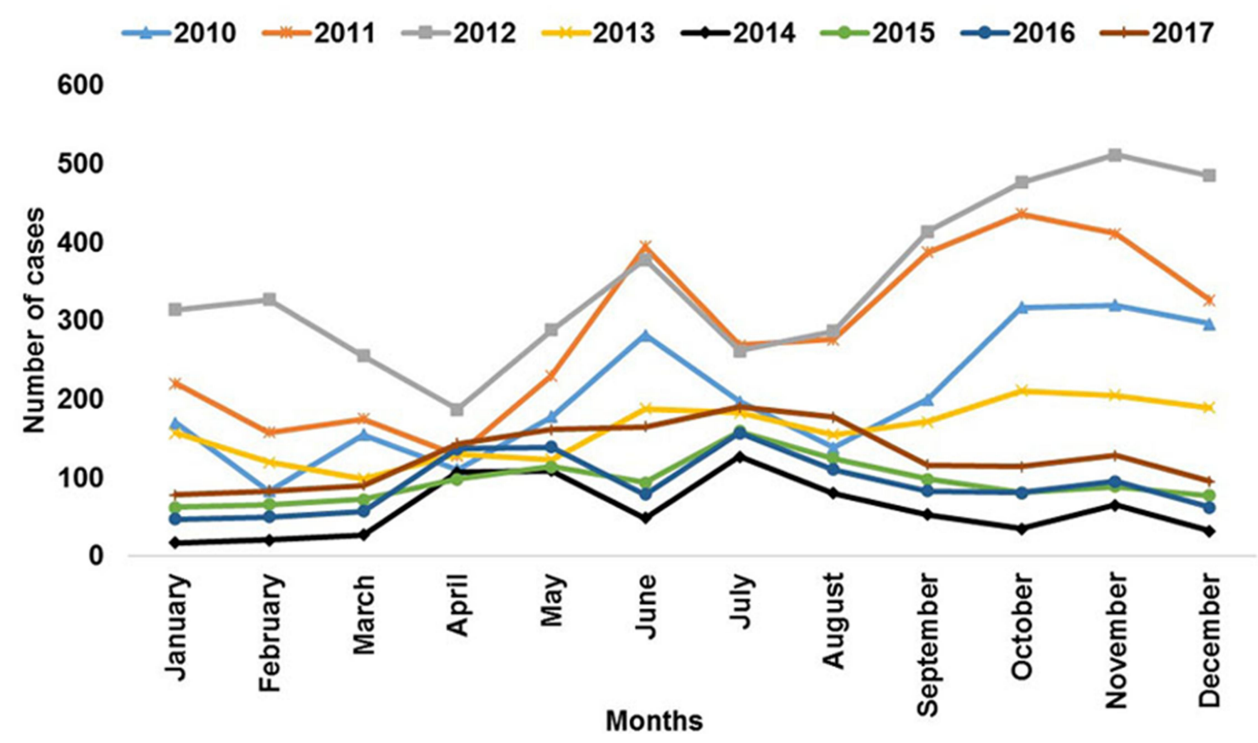

Figure 6 Seasonal distribution of malaria from 2010 to 2017 in Bale zone, Southeast Ethiopia.

and reduction in annual incidence is suggestive of good progress towards achievements of 2020 goals of Ethiopia and progressive steps of transition towards the preelimination phase set by WHO, which is $<5$ cases $/ 1,000$ populations at risk/year. ${ }^{13,14}$

Laboratory confirmed (microscopy/RDT) malaria incidence rate was $<5 / 1,000$ population each year during the study period, which is indicative of achievement of the HSDP-IV (Ethiopian Health Sector Development Plan) target. $^{14}$ Laboratory confirmed malaria cases were decreased by 53\% between 2012 and 2017, which is nearly similar with the national reduction in confirmed malaria cases by $48 \%$ between 2011 and 2016 and the $49 \%$ reduction during 2010-2016 according to the WHO malaria report of Ethiopia. ${ }^{2,6}$ This is possibly due to high coverage of LLIN and IRS at the community level, and high coverage of pregnant women and children under 5 years who slept under a net. ${ }^{5,6,12}$

Confirmation of malaria cases was increased by $42.2 \%$ during this 8 -years period, with a total of $66.7 \%$ confirmed cases during the study period. This finding is supported by a study of malaria trends conducted in the Tigray region, with increased confirmation of malaria cases by $48 \%$ between 2011/12 and 2014/15. ${ }^{15}$ Similarly, 33\% of laboratory confirmation of malaria suspected cases were reported nationally between 2011 and 2016 according to a national report. ${ }^{6}$ The overall 8 -year test positivity rate of $17.6 \%$ was nearly similar with the national report of malaria between 2011 and 2016. ${ }^{6}$ This finding implies that increased confirmation of malaria is possibly due to an increased supply of laboratory diagnostic tools and logistics. However, the trends in number of examined suspected cases of malaria were declining substantially by $68 \%$ between 2012 and 2017. The reason for temporal decline in examinations of suspected cases might be due to shortage of laboratory diagnostic tools and poor linkage of suspected cases to the laboratory for diagnosis by health care providers.

The dominant species of malaria during the study period was Plasmodium falciparum, which accounts for $81.5 \%$ of confirmed cases and is predominately reported throughout the study period. This finding is in agreement with malaria species distribution in several parts of Ethiopia, which reported the predominance of P. falciparum over $P$. vivax. ${ }^{6,10,11,15}$ Similarly, the higher proportion of P. falciparum (81.5\%) in our study is consistent with a national report of malaria indicator survey in 2015, which reported the $81.6 \%$ proportion of P. falciparum, $8.0 \%$ P. vivax nationally and the Oromia regional report of $87.5 \%$ and $12.5 \%$ proportion of P. falciparum and P. vivax, respectively. ${ }^{6}$ However, the proportion of $P$. falciparum in our study $(81.5 \%)$ was higher compared to the annual performance report of EPHI in 2014/15 (63.7\%). ${ }^{6}$ This might be due to variation between national figures compared to a single zone. It could also be due to the topography of the study area, which is that half of its surface area and the majority of woredas (12/15) in Bale zone are malaria endemic with an altitude below 2,000 meters above sea level. The predominance of $P$. falciparum at the higher proportion indicates 
the possibility of severe cases is higher and this finding was higher than a different report and publication. ${ }^{5,15,16}$

Annual death rate related to malaria was below 1/ 100,000 population at risk every year during the study period and with a mean annual death rate of 0.15 per 100,000 population per year. The finding of malaria mortality was below the national annual death rate report of WHO which was $0.5 / 100,00$ in $2014,1.4 / 100,000$ in 2015, and 1.05/100,000 in 2016 and a HSTP target of 3.3/ 100,000 by $2016 / 17$. $^{2,17}$ The remarkable reduction in mortality from malaria is possibly due to expanded use of RDT by health extension workers at lower level health facilities and scale up of ACT treatment as the first line treatment for uncomplicated $P$. falciparum and mixed infection. ${ }^{16}$ Besides this, the decrease in mortality is due to the expansion of treatment services for malaria in Ethiopia, as currently more than $90 \%$ of health facilities offer malaria treatment services and implementation of malaria control strategies at the grass root level. ${ }^{2,17}$ This finding is indicative of good progress towards achievement of the national goal set by 2020 , which is achieving near zero malaria.

The study also revealed a higher proportion of malaria cases among males (62.9\%) than females (37.1\%). The mean annual incidence of malaria for males (4.12 cases per 1,000 population) was also higher than females (3.61 per 1,000 population). This finding is consistent with the study conducted in Tigray Region, which reported a higher proportion of malaria cases $(69 \%)$ among females. ${ }^{15}$ Similarly, our finding is in line with a similar study of trend of malaria prevalence in south-west Ethiopia, ${ }^{18}$ and the findings of a national Malaria indicator survey of Ethiopia conducted in 2015. ${ }^{5}$ The higher malaria cases and incidence in males is due to the fact that males are usually engaged in outdoor activities, especially in the agricultural activities that put them at greater risk of contracting the disease. ${ }^{19,20}$ Furthermore, the peak malaria transmission often coincides with the planting and harvesting season, and the majority of malaria burden is among working adults in rural agricultural areas. ${ }^{6}$ In the study area, male adults are widely engaged in agricultural activities.

\section{Limitations of the Study}

In this study, the secondary data of surveillance were used to analyze the trends in malaria morbidity and mortality, which could be the possible limitations of the study. The actual burden of malaria in the community might be underestimated as a result of the collected data only including the health facilities in the study area. The registered data lacks some important variables, multiple age category and death record by age and gender, therefore difficult to measure the morbidity of malaria by comparing different age groups and death rate disaggregated by gender and age groups. In addition, the number of malaria deaths might be under-reported and underestimated, have simply not been detected by the health system and as malaria deaths in the community is not included. However, the present study showed the trends of malaria morbidity and mortality in the study area which provides important information to strengthen the intervention of malaria control.

\section{Conclusion}

Significant reduction in annual incidence and mortality is indicative of achievements of MDG target set for 2014/15 and suggestive of good progress towards achievements of 2020 goals of Ethiopia and progressive step of transition towards pre-elimination phase set by WHO. Reduction in morbidity and mortality achieved due to implementation of malaria prevention strategies at the community level and expanded use of RDT by HEWs and scale up of ACT treatment as the first line treatment. Although the reduction in morbidity and mortality achieved, the magnitude, incidence of malaria, and proportion of P. falciparum varies among woredas and the study identified higher possibilities of severe case occurrences. Hence, interventions should be adjusted to address P. falciparum species, and special attention should be given for the woredas with the highest proportion of $P$. falciparum. Moreover, further analytical studies which determine the reason for the high proportion of $P$. falciparum in the study area are recommended for the researchers.

\section{Data Sharing Statement}

Data will be available upon reasonable requests.

\section{Ethics Approval}

The study utilized existing secondary data of surveillance from the public health emergency management database of Bale Zonal Health Department and does not require ethical approval. Permission to use the data and conduct the study was obtained from the Bale Zone Health Department. Confidentiality of the information gathered from the surveillance database was assured and all information was used for this study only. 


\section{Acknowledgments}

We would like to acknowledge Mr Debru Bayu, PHEM focal person of Bale Zone, and Mr Abdurahman, Bale zone malaria department head, for making the data available. We would also like to acknowledge the support we got from Jimma University Department of Epidemiology, Ethiopian Public Health Institute and Ethiopian Field Epidemiology Training Program.

\section{Author Contributions}

All authors made substantial contributions to the conception and design, acquisition of data, or analyses and interpretation of data; took part in drafting the article or revising it critically for important intellectual content; agreed on the journal to which the article will be submitted; gave final approval of the version to be published; and agree to be accountable for all aspects of the work.

\section{Funding}

This research received no specific finance from any funding agency.

\section{Disclosure}

The authors declare that they have no competing interests.

\section{References}

1. Gerhard V, Pat D, David D, et al. A novel approach for modeling malaria incidence using complex categorical household data: the minimum message length (MML) method applied to Indonesian Data. Comput Ecol Softw. 2012;2(3):140-159.

2. World Health Organization. World malaria report 2017. Geneva, Switzerland: WHO; 2017.

3. Shepard DS, Ettling MB, Brinkmann U, et al. The economic cost of malaria in Africa. Trop Med Parasitol. 1991;42(3):199-203.

4. Gallup JL, Sachs JD. The economic burden of malaria. Am J Trop Med Hyg PubMed. 2001;64(1):85-96. doi:10.4269/ajtmh.2001.64.85

5. Ethiopian Public Health Institute. Ethiopia National Malaria Indicator Survey 2015. Addis Ababa, Ethiopia: Ethiopa Ministry of Health; 2016.

Infection and Drug Resistance

\section{Publish your work in this journal}

Infection and Drug Resistance is an international, peer-reviewed openaccess journal that focuses on the optimal treatment of infection (bacterial, fungal and viral) and the development and institution of preventive strategies to minimize the development and spread of resistance. The journal is specifically concerned with the epidemiology of
6. U.S. President's Malaria Initiative. Ethiopia Malaria Operational Plan FY 2018. USAID; 2018.

7. World Malaria Report. Ethiopia country profile: roll back malaria monitoring and evaluation 2005.

8. Central Statistical Agency of Ethiopia (2007). Summary and statistical report of the 2007 population and housing census. Addis Ababa, Ethiopia; 2007.

9. Federal Democratic Republic of Ethiopia, Ministry of Heath (MOH). National Malaria Guidelines Third Edition. Addis Ababa: MOH; 2012.

10. Abeku TA, van Oortmarssen GJ, Borsboom G, de Vlas SJ, Habbema JDF. Spatial and temporal variations of malaria epidemic risk in Ethiopia: factors involved and implications. Acta Trop. 2003;87(3):330-331. doi:10.1016/S0001-706X(03)00123-2

11. Deressa W, Shelleme C, Olana D. Magnitude of malaria admissions and deaths at hospitals and health centers in Oromia, Ethiopia. Ethiop Med J. 2004;42(4):237-246.

12. The Federal Democratic Republic of Ethiopia Ministry of Health $(\mathrm{MOH})$. Ethiopia National Malaria Indicator Survey 2011. Addis Ababa, Ethiopia; 2012.

13. Federal Democratic Republic of Ethiopia, Ministry of Heath (MOH). National Malaria Strategic Plan 2014-2020. Addis Ababa, Ethiopia: $\mathrm{MOH} ; 2014$

14. The Federal Democratic Republic of Ethiopia Ministry of Health (MOH). Health Sector Development Program IV (2010/11-2014/ 15). Addis Ababa, Ethiopia; 2010.

15. Gerensea H, Teklay H. Pattern and Trend of Malaria Morbidity and Mortality in Tigray Region, Ethiopia from 2011/12-2014/15. $J$ Bioanal Biomed. 2017;9(2):114-117. doi:10.4172/1948593X.1000163

16. Deribew A, Dejene T, Kebede B, et al. Incidence, prevalence and mortality rates of malaria in Ethiopia from 1990 to 2015: analysis of the global burden of diseases 2015. Malar J. 2017;16(271):1-7. doi:10.1186/s12936-017-1919-4

17. The Federal Democratic Republic of Ethiopia Ministry of Health (MOH). Health Sector Transformation Plan 2015/16-2019/20 (2008-2012 EFY). Addis Ababa, Ethiopia: MOH; 2015.

18. Sena LD, Deressa WA, Ali AA. Analysis of trend of malaria prevalence in south-west Ethiopia: a retrospective comparative Study. Malar J. 2014;13:188. doi:10.1186/1475-2875-13-188

19. Hawaria D, Getachew H, Zhong G, et al. Ten years malaria trend at Arjo-Didessa sugar development site and its vicinity. Southwest Ethiopia: a retrospective study. Malar J. 2019;18:145.

20. Karunamoorth K, Bekele M. Prevalence of malaria from peripheral blood smears examination: a 1-year retrospective study from the Serbo health center, Kersa Woreda, Ethiopia. J Infect Public Health. 2009;2:171-176. doi:10.1016/j.jiph.2009.08.005

\section{Dovepress}

antibiotic resistance and the mechanisms of resistance development and diffusion in both hospitals and the community. The manuscript management system is completely online and includes a very quick and fair peerreview system, which is all easy to use. Visit http://www.dovepress.com/ testimonials.php to read real quotes from published authors. 